\title{
Endoscopic Ultrasound-Guided Liver Biopsies: Is the Future Here Yet?
}

\author{
Ihab I. El Hajj ${ }^{1,2,3}$ and Mohammad Al-Haddad ${ }^{1}$ \\ ${ }^{1}$ Division of Gastroenterology and Hepatology, Department of Medicine, Indiana University School of Medicine, Indianapolis, IN, USA, \\ ${ }^{2}$ Division of Gastroenterology, St. George Hospital University Medical Center, University of Balamand, Beirut, Lebanon, ${ }^{3}$ Division of \\ Gastroenterology, Clemenceau Medical Center Affiliated with Johns Hopkins International, Beirut, Lebanon
}

See “Comparison of Endoscopic Ultrasound Biopsy Needles for Endoscopic Ultrasound-Guided Liver Biopsy" by Armen Eskandari, Patrick Koo, Heejung Bang, et al., on page 347-352.

See "Endoscopic Ultrasound-Guided Liver Biopsy Using a Core Needle for Hepatic Solid Mass" by Hyung Ku Chon, Hee Chan Yang, Keum Ha Choi, et al., on page 340-346.

Endoscopic ultrasound-guided fine needle aspiration (EUSFNA) has been proven as an effective modality for sampling of gastrointestinal and liver lesions. ${ }^{1,2}$ The sampling needle type represents one of the several factors that affect the EUS tissue acquisition (EUS-TA) outcomes. ${ }^{3,4}$ In the last few years, the progressive replacement of EUS-FNA with EUS guided-fine needle biopsy (EUS-FNB) was noted due to the introduction of several specially designed needles that provide core tissue samples that allow for histological assessment. The EchoTip ProCore (Cook Medical, Winston-Salem, NC, USA) needle has a reverse bevel design and the EZ shot 3 plus needle (Olympus America Inc., Lombard, IL, USA) has a flexible coil sheath that allows for precise and smooth penetration into the target lesion. The SharkCore (Medtronic, Minneapolis, MN, USA) needle has a fork-tip design with 2 leading sharp tips and the Acquire needle (Boston Scientific Co., Natick, MA, USA) has a Franseen tip design with 3 symmetrical cut-

Received: June 26, 2019 Accepted: June 27, 2019

Correspondence: Mohammad Al-Haddad

Division of Gastroenterology and Hepatology, Department of Medicine, Indiana University School of Medicine, 550 N. University Blvd, Suite 4100, Indianapolis, IN 46202, USA

Tel: +1-317-944-7896, Fax: +1-317-948-8144, E-mail: moalhadd@iu.edu ORCID: https://orcid.org/0000-0003-1641-9976

(c) This is an Open Access article distributed under the terms of the Creative Commons Attribution Non-Commercial License (http://creativecommons.org/ licenses/by-nc/3.0) which permits unrestricted non-commercial use, distribution, and reproduction in any medium, provided the original work is properly cited. ting surfaces. ${ }^{5}$ When compared with conventional ultrasound and computed tomography scan, EUS showed superiority in the detection and sampling of liver lesions. In a recent review of 9 published studies ( 6 prospective, 2 retrospective, and 1 observational), the diagnostic accuracy of tissue sampling of liver lesions via EUS-FNA and/or FNB ranged from $80 \%$ to $100 \%{ }^{6}$

In this issue, Eskandari et al. ${ }^{7}$ compared the performance of 6 needles with different gauges (SharkCore 19 and $22 \mathrm{G}$, Acquire 19 and 22 G, EZ Shot 3 Plus 19 G, and EchoTip ProCore $20 \mathrm{G}$ ) for EUS-guided liver biopsy on a freshly harvested bovine liver. All the 19 and $20 \mathrm{G}$ needles yielded similar mean numbers of complete portal tracts (CPTs) and were significantly superior to the SharkCore $22 \mathrm{G}$ needle. The outcomes with the large bore needles were significantly superior to that with the 22 G SharkCore needle. No statistically significant difference in the total specimen length was found among the three $19 \mathrm{G}$ needles and the $20 \mathrm{G}$ needle tested. The performance of the two $22 \mathrm{G}$ needles was similar (number of CPTs, mean fragment length, and mean specimen length).

In this same issue, Chon et al. ${ }^{8}$ studied the safety, efficacy, and outcomes of EUS-FNB using a core needle (EchoTip ProCore HD ultrasonography-guided biopsy needle) in 58 hepatic solid masses (46 metastatic and 16 non-metastatic lesions). The metastatic lesions included 38 metastatic pancreatic cancer lesions, 4 metastatic ampullary cancer lesions, and 4 meta- 
static lung cancer lesions. The non-metastatic lesions included 9 cholangiocarcinomas and 3 hepatocellular carcinomas. The diagnostic accuracy was $89.7 \%$. The sensitivity and specificity were $89.7 \%$ and $100 \%$, respectively. The analysis of factors that could affect the diagnostic accuracy of EUS-FNB for hepatic solid masses revealed no significant associations. The factors assessed included the number of needle passes, needle gauge, needle approach route (transgastric or transduodenal), and mass size.

Both studies have several limitations. The first study was performed on an ex vivo liver model; thus, adverse events could not be evaluated, and a real-time tissue evaluation may be affected by anatomical and technical variations. The second study was retrospective, with a relatively small sample size and possibly underreported adverse events.

At our facility and on the basis of the increasing evidence supporting the use of FNB for sampling solid lesions and performing targeted biopsies, we have gradually adopted FNB as a replacement for FNA for the sampling of solid lesions. This approach has the advantages of fewer number of passes and shorter overall procedure time. ${ }^{4}$ We strongly believe that future needle platforms will adopt an improved tip design for better access and enhanced core retention to optimize EUSTA. The main question remains whether the available literature provides enough evidence to support the use of EUSFNA and/or EUS-FNB for liver lesions as a replacement for percutaneous liver biopsy, which has been considered the gold standard for many years. Further studies are needed to address this concern.

Conflicts of Interest

The authors have no financial conflicts of interest.

\section{REFERENCES}

1. Wani S, Shah RJ. EUS-guided tissue acquisition: do we need to shoot for a "core" to score? Gastrointest Endosc 2016;84:1047-1049.

2. El Hajj II, Al-Haddad M. Endoscopic ultrasound-guided tissue acquisition of pancreatic masses. Tech Gastrointest Endosc 2018;20:30-38.

3. Al-Haddad M. Fine-needle biopsy sampling under EUS guidance: is one needle tip really better than the other? Gastrointest Endosc 2018;87:1163.

4. El Haij II, Al-Haddad M. EUS-FNA giving way to fine-needle biopsy: is it time to retire your old trusted needles? Gastrointest Endosc 2018;87:1439-1442

5. El Hajj II, Wu H, Reuss S, et al. Prospective assessment of the performance of a new fine needle biopsy device for EUS-guided sampling of solid lesions. Clin Endosc 2018;51:576-583.

6. Ichim VA, Chira RI, Mircea PA. Diagnostic yield of endoscopic ultrasound-guided biopsy of focal liver lesions. Med Pharm Rep 2019;92:1520.

7. Eskandari A, Koo P, Bang H, Gui D, Urayama S. Comparison of endoscopic ultrasound biopsy needles for endoscopic ultrasound-guided liver biopsy. Clin Endosc 2019;52:347-352.

8. Chon HK, Yang HC, Choi KH, Kim TH. Endoscopic ultrasound-guided liver biopsy using a core needle for hepatic solid mass. Clin Endosc 2019;52:340-346 